\title{
Imagen de la marca Perú y generación de valor para el turista de Lima Metropolitana, Perú
}

\author{
Peru's brand image and the generation of value for tourists in \\ Metropolitan Lima, Peru
}

\author{
Karla Mabel Cueva Gamboa ${ }^{1}$, Renzo Gianfranco Sánchez Chepe ${ }^{1}$, Jorge Alberto \\ Vargas-Merino $^{1,2}$ \\ ${ }^{1}$ Universidad Privada Norbert Wiener \\ ${ }^{2}$ Universidad Privada del Norte
}

\begin{abstract}
Resumen
Desde que se creó la marca Perú en el año 2011, esta ha tenido un crecimiento considerable debido a que Promperú, su principal embajador, impulsa la marca buscando generar orgullo, identidad y autoestima, y resaltar su diferenciación de otros países, para lo cual se procura también que existan propuestas turísticas que logren generar valor dentro de toda la cadena, es decir, una experiencia turística integral. La presente investigación tuvo como objetivo general determinar cómo la imagen de la marca Perú influye en la generación de valor para el turista de Lima Metropolitana, Perú, en el año 2020. Se trabajó con un método hipotético-deductivo, un enfoque cuantitativo, tipo de investigación básica de nivel explicativo y un diseño no experimental de corte trasversal. La muestra objeto de estudio fue de 228 turistas nacionales, el instrumento utilizado fue el cuestionario, el cual obtuvo un alfa de Cronbach de 0,82, por lo cual existe una alta confiabilidad estadística y una validación de contenido del $80 \%$ por parte de los evaluadores expertos. De manera general, se concluye que existe una influencia significativa entre la imagen de la marca Perú y la generación de valor para el turista de Lima Metropolitana en el 2020, ya que se obtuvo un nivel de significancia de $0,00<0,05$ en el estadístico de contraste Chi cuadrado. Esto es, cuando la imagen de la marca Perú se gestiona adecuadamente tiene una influencia directa y significativa en la generación de valor percibido por el turista. La generación de valor es explicada por la gestión de la imagen de la marca Perú de manera significativa.
\end{abstract}

Palabras clave: Imagen de la marca Perú, generación de valor, gestión de la marca, reputación de la marca, infraestructura turística.

Fecha de envío: 20/06/2021

Fecha de aceptación: 20/08/2021 


\begin{abstract}
Since the Peru brand was created in 2011, it has had considerable growth due to the fact that Promperú, its main ambassador, promotes the brand seeking to generate pride, identity and self-esteem, and highlight its differentiation from other countries, for which it is It also ensures that there are tourist proposals that manage to generate value within the entire chain, that is, a comprehensive tourist experience. The present investigation had as general objective to determine how the image of the Peru brand influences the generation of value for the tourist of Metropolitan Lima, Peru, in the year 2020. We worked with a hypothetical-deductive method, a quantitative approach, type of explanatory-level basic research and a non-experimental cross-sectional design. The sample under study was 228 national tourists, the instrument used was the questionnaire, which obtained a Cronbach's alpha of 0.82 , for which there is high statistical reliability and a content validation of $80 \%$ by expert evaluators. In general, it is concluded that there is a significant influence between the image of the Peru brand and the generation of value for the tourist of Metropolitan Lima in 2020, since a level of significance of $0.00<0.05$ was obtained in the Chi-square test statistic. That is, when the image of the Peru brand is properly managed, it has a direct and significant influence on the generation of value perceived by tourists. The generation of value is explained by the management of the image of the Peru brand in a significant way.
\end{abstract}

\title{
Keywords: Peru brand image, value generation, brand management, brand reputation, tourism infrastructure.
}

\section{Introducción}

Hace algunos años, la observación del uso de conceptos y recursos del marketing hacía referencia exclusivamente a los bienes y servicios con el único fin de llevarlos al terreno de la promoción de países. El término "Marca País" nace a partir de la necesidad de los gobiernos de instaurar su propia identidad como nación, frente a los mercados mundiales (Ricaurte y Juan, 2016).

Latinoamérica tiene un gran camino por recorrer respecto a la Marca País, ya que de los 75 paises que fueron evaluados en el Global Top 75, ningún país latinoamericano se encontró dentro de los 30 primeros. Japón fue el país que encabezó el ranking y mantuvo su primer lugar desde el año 2014, a diferencia de otros países como Estados Unidos que retrocedieron cuatro lugares o Alemania que retrocedió tres lugares. En el puesto 36 se encuentra Argentina y Perú ocupa el puesto 37. Algunos de los factores que influyen para que los turistas elijan a un país para poder visitarlo y convertirlo en uno de sus lugares favoritos es que el país debe poseer una identidad y una reputación fuerte, mostrar una calidad de vida favorable, poseer diversidad para conocer atractivos naturales, entre otros factores, con el fin de poseer una ventaja competitiva frente al resto de marcas país (FutureBrand y Country Brand Index, 2019).

En el contexto nacional, Gestión (2019) 
señala que la marca Perú es un signo que busca impulsar las exportaciones, conquistar el turismo y atraer las inversiones, además de que es un signo que contribuye a realizar marketing peruano al ofrecer todo lo bueno que se puede ofrecer como país. Es por ello que se pretende proteger la marca Perú en los diversos países del mundo, ya que muchas personas o empresas inescrupulosas hacen uso de ella sin antes cumplir los estándares de calidad que se requieren para poder ser una marca representativa.

En la gestión de los productos turísticos el valor es un componente esencial, pues, en general, no existe una necesidad perentoria de utilizar o consumir productos turísticos, tomando en consideración que la puesta en valor de los productos turísticos requiere de un mayor esfuerzo de gestión que el requerido para los productos y servicios de primera necesidad (Secretaría General de Turismo, 2007).

Por otro lado, el problema de la informalidad en el turismo genera una competencia desleal para las agencias de viaje, ya que, en el 2016, el $65 \%$ y $70 \%$ de las empresas del rubro turista eran informales. Esta situación es un problema que se deberá de resolver con mucha responsabilidad por parte del país, ya que estas empresas informales son las creadoras de la mala imagen frente a los turistas que están en camino a generar valor a la marca nacional (Aguilar y Herrera, 2018).

Mori (2018) nota que la generación de valor en el turista de Lima se ha transformado en un tema de preocupación por parte del gobierno y de las entidades del sector turismo. Es un compromiso de los órganos competentes como MINCETUR,
PROMPERÚ, entre otros. Si estas entidades no generan confianza en los ciudadanos, no podrán generar un valor aceptable dentro de este sector. Es por ello que nace la preocupación por el orden, coordinación y gestión adecuadas de la actividad turística, bajo un criterio de ciudad sustentable, para lo cual se toman como bases las fortalezas y debilidades que la gestión turística pueda lograr.

Así, surge una gran interrogante de investigación: ¿de qué manera la imagen de la marca Perú influye en la generación de valor para el turista de Lima Metropolitana en el año 2020? La hipótesis que proponemos es que la imagen de la marca Perú influye de manera significativa en la generación de valor para el turista de Lima Metropolitana en el año 2020. De forma específica proponemos tres hipótesis: 1) La imagen de la marca Perú influye en la oferta y demanda turística; 2) La imagen de la marca Perú influye en la calidad de vida del turista; y 3) La imagen de la marca Perú influye en la infraestructura turística. El objetivo de la investigación fue determinar de qué manera la imagen de la marca Perú influye en la generación de este valor.

\subsection{Antecedentes}

En una investigación colombiana realizada por Ayala y Reyes (2019), se tuvo como objetivo el analizar la evolución y el comportamiento de la marca País Colombia, para lo cual se tuvo como relación las estrategias de mercadeo en los años 20152018; se siguió un enfoque cualitativo, tipo descriptivo; se realizó con una muestra compuesta por 105 artículos, de los 
cuales fueron utilizados 62 para el análisis; se realizó desde filtros delimitados por idiomas y accesibilidad. Se llegó a la conclusión de que la marca país acrecienta la competitividad y posicionamiento de la imagen de un país a nivel internacional y puede ser utilizada a favor a la hora de invertir en un país, conocer o simplemente cambiar el pensamiento que se tiene sobre determinado país (Ayala y Reyes, 2019).

En el artículo científico Marca país (2010-2014): Ecuador Ama la Vida y su incidencia en la matriz productiva y el plan del Buen Vivir, realizado en Ecuador por Ferrán, Naranjo y Marrero (2016), se tuvo como objetivo realizar una reflexión sobre la campaña promocional de destino marca país "Ecuador ama la vida". Se siguió un enfoque cualitativo, con una investigación bibliográfica y se utilizó el instrumento de la entrevista semiestructurada con expertos, para finalmente concluir que la campaña turística "Ecuador ama la vida" tuvo un trabajo de éxito para lograr su posicionamiento como marca país, logrando conseguir ser un instrumento de la diplomacia pública (Ferrán, Naranjo y Marrero, 2016).

En otra investigación se tuvo como objetivo general el conocer si el uso de la marca Perú incentivó a la oferta turística en el año 2018. La investigación cuenta con un enfoque cualitativo, con un diseño de carácter inductivo e interpretativo y se trabajó con un cuestionario. Se concluye que, aunque no hay evidencias significativas sobre el uso de la marca para generar un incremento en el turismo nacional, se puede observar el incremento de los turistas nacionales, lo cual se debería al uso de campañas particulares que promocionan a la marca Perú (Cáceres y Delgado, 2019).

En la investigación titulada Marca Ciudad para mejorar la promoción turística del distrito de Tarapoto, año 2017 se tuvo como objetivo diseñar la marca cuidad para mejorar la promoción turística del distrito de Tarapoto. La investigación fue cuantitativa, no experimental, tuvo una población de 376416 y una muestra de 384 visitantes, así como se empleó el instrumento del cuestionario. Se concluyó que la marca Perú es importante debido a que se fortalece con la imagen e identidad, por lo cual el país puede contar con la ayuda de esta herramienta que tiene como objetivo el poder fomentar el turismo a nivel nacional (Cotrina, 2018).

En la tesis de Luyo (2019) que lleva por título La marca Perú y su incidencia en la promoción de la oferta turística en nuestro país durante el año 2017; analizando su posicionamiento y consolidación en el mercado internacional se tuvo como objetivo establecer el nivel de incidencia de la marca Perú en la promoción de las ofertas turísticas de nuestro país, para lo cual se analizó su posicionamiento y consolidación en el mercado internacional. Esta fue una investigación cualitativa, no experimental, tuvo una muestra de tres expertos con 5 años de experiencia, y un instrumento de recolección de datos a través de entrevistas. En ella se concluye que la oferta turística motiva a los turistas, por lo que es fundamental poder brindar mejores posibilidades al turista, para lo cual se daría un valor agregado a la promoción turística con estrategias y los suficientes recursos para poder obtener mejores resultados (Luyo, 2019).

En la investigación titulada El proce- 
so de creación de "Más Peruano Que" y de sus piezas publicitarias: una campaña de Marca Perú basada en generar orgullo peruano, realizada por Sánchez (2019), se tuvo como objetivo conocer las estrategias de "Más peruano Que" en su proceso de creación y sus piezas. Fue una investigación cualitativa, se trabajó con entrevistas, focus group y encuestas, se tuvo como conclusión que la idea de marca Perú se planteó en el año 2009 al tener como objetivo el poder desarrollar el crecimiento de la autoestima como herramienta de promoción. Sin embargo, en el año 2011 se lanza la campaña marca Perú. Además, Promperú convocó en el 2014 a una licitación paracontratar una agencia con el objetivo de despertar el orgullo peruano. Esto resultóen iniciativas tales como "Perú Nebraska", y se tuvo como mensajes el "Yo valoro que soy, porque la gente valora lo que yo tengo" y el "Mas Peruano Que" (Sánchez, 2019).

Caycho y Prieto (2018) presentan una investigación que tuvo como objetivo analizar si el efecto de la marca Perú favoreció al sector turismo en Lima durante los años 2014 - 2017. Es una investigación descriptiva que utilizó como técnicas la encuesta y el análisis de documental, con el cuestionario y fichas de resumen como sus instrumentos. La conclusión es que, en general, la marca Perú ha tenido un impacto positivo sobre el turismo en el distrito de Lima, pues se encontróque las personas se sienten motivadas e interesadas en conocer los lugares céntricos y turísticos de Lima, ya que a los visitantes les llama mucho la atención la cultura, el arte, y las historias vividas que suelen contar las agencias de viaje para proporcionar un buen servi- cio al turista.

En la investigación La supervivencia de las agencias de viaje y turismo: gestión del marketing digital como estrategia para la atracción de clientes turistas realizada por Aguilar y Herrera (2018) se tuvo como objetivo analizar la influencia de los factores de éxito para la atracción de viajeros nacionales. Fue una investigación mixta, experimental, y tuvo como instrumento de recolección de datos las encuestas. Se concluye que la generación de valor se da mediante los medios digitales, sobre todo las páginas web y las redes sociales, las cuales son de gran consideración por los turistas, sobre todo de aquellos que manejan un perfil y tienen interacción digital (Aguilar y Herrera, 2018).

La marca Perú es una herramienta que busca fomentar el turismo y difundir una propuesta de valor del país con eficacia (Promperú, 2018).

La marca país, por su parte, tiene una orientación cultural que se observa en la identidad nacional, de acuerdo con una dinámica de lucha y de negociación sobre los significados individuales y colectivos (Matta, 2012).

La marca Perú conforma una estrategia de promoción del país y tiene como reto el poder destacar, llamar la atención y transmitir una propuesta brillante. Sus pilares son el amor por la cultura, el amor y deseo por un país con enfoque ambiental natural. La marca Perú ha pulido un lazo inalterable, un vínculo que forma parte del patrimonio y potencia nuestra cultura (Mendivez, 2020).

Desde el punto de vista estratégico de una empresa, la generación de valor es consecuencia de un mecanismo de posicio- 
namiento y diferenciación de una marca frente al resto (Carballa, 2016).

Mori (2018) refiere que la generación de valor busca que los turistas vivan experiencias satisfactorias en el momento que consumen algún servicio. Es por ello que cada una de las fases del "momento de la verdad" en el servicio debe ser extremadamente cuidadosa y eficiente. La suma de todos estos valores genera un mayor valor añadido en el servicio final.

Para Aguilar y Arteaga (2017), la generación de valor en términos financieros es una inversión o transacción que es capaz de retornar a su mismo lugar con un monto adicional de dinero al que se tenía inicialmente. Visto en contexto, solo habrá valor cuando haya retorno de la inversión y cuando la cadena turística perciba ingresos por encima de su inversión.

Sulz (2019) menciona que la gestión de marca tiene como objetivo hacer conocido y deseado un determinado producto o servicio, para lo cual genera una imagen positiva en la mente de los consumidores y crea una conexión duradera con las personas.

La reputación de marca es la representación y el sentimiento con el que se le asocia, y se construye mediante la experiencia personal, los diversos puntos de vista y lo que se dice de la marca (Maram, 2018).. La reputación de marca se tiende a consolidar en la memoria colectiva cuando se establece una ventaja competitiva (Suarez, 2019).

El conocimiento de marca es la capacidad de poder distinguir algún producto o servicio del resto, llegando al nivel de conciencia y no solo reconocer alguna imagen (Velasquez, 2019). Esto es importante pa- ra poder crear un valor, ya que se crea un efecto diferencial; sin embargo, la fortaleza del nodo de la marca o la huella en la memoria es en donde finalmente se mide la capacidad del consumidor para poder identificarla en diversas condiciones (Alba, 2017).

Según Romero (2016), la demanda turística tiene lugar cuando un individuo o un grupo de personas deciden viajar y se desplazan, ya sea fuera o dentro de un país, por un determinado tiempo consecutivo menor a un año, con la finalidad de pasar un momento de ocio, por negocios u otros motivos. Asimismo, puede verse en un grupo de consumidores turísticos que deciden movilizarse para consumir productos o servicios en el lugar de destino. Existen dos tipos de demanda turística: la demanda nacional, que representa a las personas que se movilizan dentro del territorio nacional, y la demanda internacional, en la que los turistas deciden viajar fuera de su país de origen. Por otro lado, el autor define a la oferta turística como un conjunto de productos turísticos y servicios que están frente al usuario para que pueda disponer de ellos. La oferta turística está compuesta por recursos turísticos, infraestructuras y empresas turísticas.

La oferta turística es el conjunto de productos turísticos y servicios que se encuentran a disposición de las personas que realizan turismo en un tiempo determinado, con el fin de que estos puedan consumir y disfrutar, mientras que la demanda turística es el proceso en el que las personas toman la decisión constante de poder desplazarse. Notemos, sin embargo, que esta decisión no solo depende de factores económicos, sino también de facto- 
res como los psicológicos, físicos y éticos (Sancho, 2016).

Para Robles, Rubio, De la Rosa y Nava (2016), la calidad de vida es la sensación de bienestar que las personas pueden experimentar y es la suma de una serie de sensaciones subjetivas. La calidad de vida está formada por la habilidad de realizar actividades en el día a día que muestran el buen estado físico psicológico y social, y la satisfacción plena.

La calidad de vida está representada por un punto de satisfacción de las necesidades básicas como alimento, trabajo, vivienda, salud, entre otros factores para un grupo de individuos. Por tanto, existe una sensación percibida de bienestar emocional, salud, seguridad, trabajo, entre otros (Ardila, 2003).

En cuanto a infraestructura turística, esta se define como el conjunto de elementos o servicios que se consideran como inevitables para que una organización funcione o para que una actividad se pueda desarrollar de una forma efectiva (Mero, 2015).

La infraestructura turística es considerada de suma importancia para el desarrollo de los recursos turísticos, ya que esto permite que la actividad turística se desarrolle con total normalidad y en las mejores condiciones. Es por ello que debe de estar siempre a disposición de todas las personas que conforman una comunidad, ya sean visitantes o personas que viven en este lugar, pues la función principal es satisfacer las necesidades de una ciudad (Arce y Bustamente, 2016).

Se define a la infraestructura turística como el conjunto de bienes y servicios con los que un país cuenta para poder hacer frente a sus estructuras sociales y productivas, y, como consecuencia, tener el desarrollo turístico. Además, esta contribuye a la creación de estructuras fuertes para poder lograr un desarrollo turístico (Angulo, 2019).

\section{Materiales y método}

Esta investigación siguió el método hipotético-deductivo y un enfoque cuantitativo, el mismo que contiene un conjunto de procesos ordenados y secuenciales probatorios. Cada etapa es la causa de la siguiente, por lo que no se debe de saltar ninguna etapa dada su vital importancia. Se deben medir las variables a través de métodos estadísticos para, finalmente, establecer las conclusiones pertinentes en relación con las hipótesis planteadas (Hernández, Fernández y Baptista, 2014).

El tipo de investigación es básica, ya que tiene como propósito la obtención de nuevos conocimientos, con lo cual se aumenta el entendimiento científico sin un fin práctico inmediato (Escudero y Cortéz, 2018). Fue de nivel explicativo, de diseño no experimental y de corte transversal, es decir, no se varía intencionalmente la variable para medir su efecto sobre otra, sino que se visualizan los fenómenos tal como estos se presentan en un contexto natural (Mousalli, 2015).

La población que fue objeto de estudio en esta investigación estuvo constituida por 4'335,523 turistas limeños que viajan al interior del país, según reporte de Promperú.

La muestra estadística se seleccionó de manera aleatoria, se tuvo como finalidad 


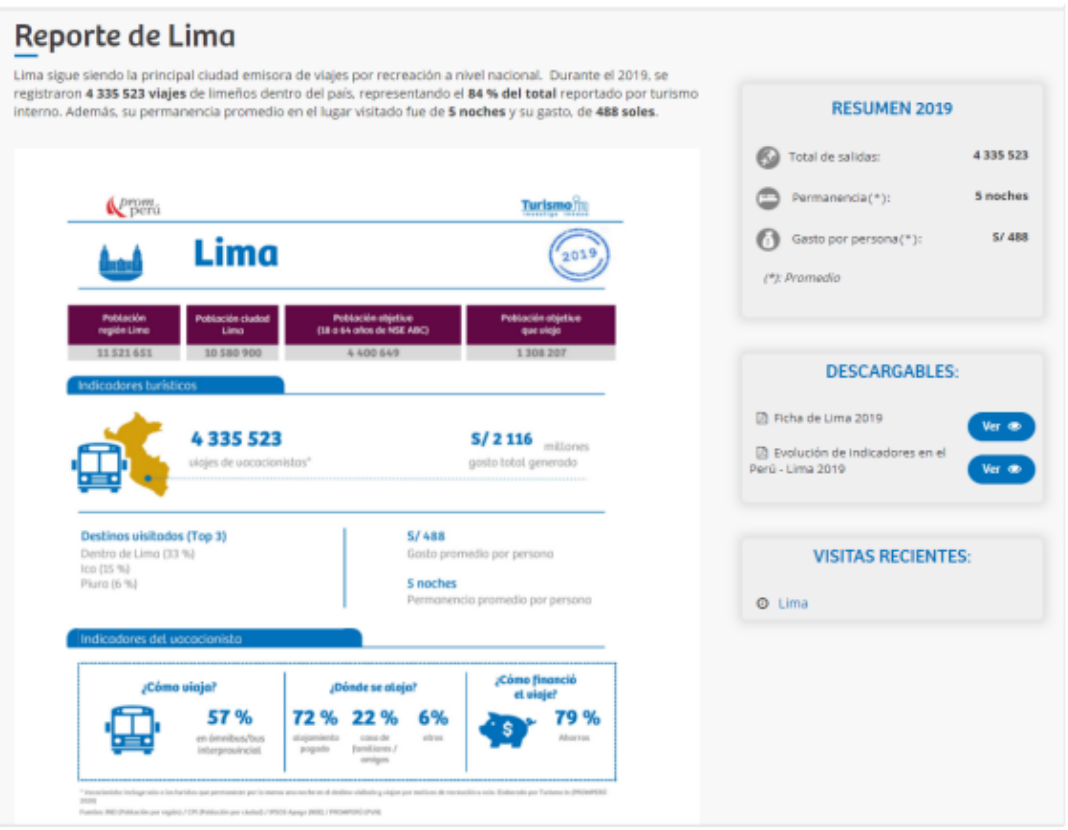

Figura 1: Reporte de número de turistas que viajan al interior del país.

conseguir los resultados válidos hacia un universo total de turistas, teniendo unos límites de error y posibilidades en la que se pueda decidir (Roldan y Fallechi, 2018). Se aplicó la fórmula probabilística para población finita cualitativa, y se obtuvo 228 turistas a ser encuestados.

El muestreo fue aleatorio simple, es decir, se tiene el aval de que todas las personas que están dentro de la población de estudio deben de tener la misma posibilidad de ser incluidas dentro de la muestra (Otzen y Manterola, 2017).

La técnica utilizada fue la encuesta; y el instrumento, el cuestionario, el cual fue objeto de validación de contenido por expertos, los mismos que analizaron cada una de las preguntas obteniendo un $\mathrm{V}$ de Aiken de 0.82. Esto muestra que esta in- vestigación tiene un $82 \%$ de validez y, en cuanto a la confiabilidad, con un alfa de Cronbach de 0.738 para la variable Imagen de la marca Perú, y un 0.656 para la variable de generación de valor. Al ser mayores a 0.60 , se tendrá como consecuencia un instrumento confiable.

\section{Resultados}

A continuación, se destacan los resultados estadísticos para las variables de estudio.

La sig. $<0.05$, por tanto, se acepta la Ha, es decir, la imagen de la marca Perú si influye de manera significativa en la generación de valor para el turista de Lima Metropolitana 2020.

La sig $<0.05$, por tanto, se acepta la 
Tabla 1: nfluencia de la marca Perú en la generación de valor para el turista de Lima Metropolitana

\begin{tabular}{llll}
\hline & Valor & df & $\begin{array}{l}\text { Significación } \\
\text { asintótica (bilate- } \\
\text { ral) }\end{array}$ \\
\hline Chi-cuadrado de Pearson & $41,516 \mathrm{a}$ & 4 &, 000 \\
Razón de verosimilitud & 36,202 & 4 &, 000 \\
Asociación lineal por lineal & 28,698 & 1 &, 000 \\
N de casos válidos & 228 & & \\
0 casillas (0,0\%) han esperado un recuento menor que 5. El re- \\
cuento mínimo esperado es 6,16. \\
\hline \multicolumn{4}{c}{ Fuente: Base de datos IBM y SPSS }
\end{tabular}

Tabla 2: Influencia de la marca Perú en la oferta y demanda turística de Lima Metropolitana

\begin{tabular}{llll}
\hline & Valor & df & $\begin{array}{l}\text { Significación } \\
\text { asintótica (bilate- } \\
\text { ral) }\end{array}$ \\
\hline Chi-cuadrado de Pearson & $46,973 \mathrm{a}$ & 4 &, 000 \\
Razón de verosimilitud & 40,058 & 4 &, 000 \\
Asociación lineal por lineal & 34,802 & 1 &, 000 \\
N de casos válidos & 228 & & \\
a. 0 casillas (0,0 \%) han esperado un recuento menor que 5. El \\
recuento mínimo esperado es 5,05.
\end{tabular}

Fuente: Base de datos IBM y SPSS

Ha, es decir que la imagen de la marca Perú si influye de manera significativa en la oferta y demanda turística de Lima Metropolitana, 2020.

La sig $>0.05$, es decir, se acepta el Ho, la imagen de la marca Perú no influye de manera significativa en la calidad de vida del turista de Lima Metropolitana, 2020.

La sig $<0.05$, esto es, se acepta la Ha, por tanto, la imagen de la marca Perú sí influye de manera significativa en la infraestructura turística.

\section{Discusión}

Se comprobó que existe una influencia significativa entre la imagen de la marca Perú y la generación de valor en el turista de Lima (sig. < 0.05). Esto indica que la generación de valor es explicada por la imagen de la marca Perú, y hacia ello deben apuntar las estrategias de éxito. Estos resultados guardan relación con la investigación de Cotrina (2018), quien, en su trabajo realizado en el distrito de Tarapoto, determinó que existen niveles altos en la 
Tabla 3: Influencia de la marca Perú en la calidad de vida del turista de Lima Metropolitana

\begin{tabular}{llll}
\hline & Valor & df & $\begin{array}{l}\text { Significación } \\
\text { asintótica (bilate- } \\
\text { ral) }\end{array}$ \\
\hline Chi-cuadrado de Pearson & $5,739 \mathrm{a}$ & 4 &, 220 \\
Razón de verosimilitud & 5,464 & 4 &, 243 \\
Asociación lineal por lineal & 4,011 & 1 &, 045 \\
N de casos válidos & 228 & & \\
a. 0 casillas (0,0\%) han esperado un recuento menor que 5. El \\
recuento mínimo esperado es 7,58.
\end{tabular}

Fuente: Base de datos IBM y SPSS

Tabla 4: Influencia de la marca Perú en la infraestructura turística de Lima Metropolitana

\begin{tabular}{llll}
\hline & Valor & df & $\begin{array}{l}\text { Significación } \\
\text { asintótica (bilate- } \\
\text { ral) }\end{array}$ \\
\hline Chi-cuadrado de Pearson & $33,117 \mathrm{a}$ & 4 &, 000 \\
Razón de verosimilitud & 27,522 & 4 &, 000 \\
Asociación lineal por lineal & 20,040 & 1 &, 000 \\
N de casos válidos & 228 & & \\
a. 0 casillas (0,0\%) han esperado un recuento menor que 5. El \\
recuento mínimo esperado es 5,53.
\end{tabular}

Fuente: Base de datos IBM y SPSS

percepción hacia la marca ciudad en Tarapoto, respecto al cual los turistas mencionan que es un lugar que da confianza, por lo que las experiencias son gratificantes.

Esto también tiene semejanza con la investigación de Caycho y Prieto (2018), en donde se logró determinar que la marca Perú sí tiene un efecto positivo en el sector turismo y las personas se sienten motivadas a poder descubrir diversos lugares y centros de interés.

En la hipótesis especifica 1 se corroboró que la imagen de la marca Perú sí influye significativamente sobre la oferta y demanda turística. Estos resultados tienen similitud con los obtenidos por Cáceres y Delgado (2019), donde se determinó una relación positiva entre la imagen de la marca Perú y la oferta y demanda turística. Asimismo, también se tiene una semejanza con la investigación realizada por Aguilar \& Herrera (2018), en donde la incidencia de ambas variables es significativa y los turistas tienen interés por las ofertas generadas en las redes sociales.

En la hipótesis 2 se pudo compro- 
bar que no existe influencia (significancia $=.220>0.05)$ entre la imagen de la marca Perú y la calidad de vida. Estos resultados no tienen similitud con los obtenidos por Ferrán, Naranjo, \& Marrero (2016) en Ecuador, quienes sí determinaron la importancia de la marca Perú para el crecimiento económico y, por ende, la mejora de la calidad de vida de la población. Tampoco se halla vinculación con los resultados de Ayala y Reyes (2019), ya que la marca país en Colombia buscaba no solo enfocarse en su mejoramiento en el exterior, sino también cambiar el concepto que los mismos colombianos tenían sobre su país. De esta manera, se contribuyó a que nuevas empresas del sector privado sumaran sus esfuerzos y, a través de sus productos, impulsaran la marca país y también el potencial de Colombia a nivel comercial, con lo cual se obtuvieron mayores ingresos que fortalecieron las acciones en bien de la sociedad colombiana y sus mejoras en la calidad de vida.

En la hipótesis especifica 3 se comprobó que la imagen de la marca Perú sí influye de manera significativa en la infraestructura turística. Estos resultados tienen similitud con los obtenidos por Sanchez (2019), quien menciona la importancia de constituir y fortalecer diversos pilares de la cadena turística para que así se logre un incremento sustancial de visitas, mayor consumo de productos y servicios. A su vez, también se tiene una semejanza con una investigación realizada por Luyo (2019) sobre la marca Perú y la atracción de turistas, teniendo como eje central el fortalecimiento integral de las diversas infraestructuras turísticas para satisfacción del turista, una cuestión de creciente im- portancia en los últimos años, ya que determina el posicionamiento de la oferta en el país en un entorno competitivo.

\section{Conclusiones}

Se determinó que existe una influencia significativa entre la imagen de la marca Perú y la generación de valor para el turista de, Lima Metropolitana en el año 2020, ya que se obtuvo como resultado un nivel de significancia de $0,00<0,05$. Es decir, cuando la imagen de la marca Perú se gestiona adecuadamente, esta tiene una influencia directa y significativa en la generación de valor percibido por el turista.

Se identificó una influencia significativa entre la imagen de la marca Perú y la oferta y demanda turística para el turista de Lima Metropolitana en el año2020 , pues se obtuvo un nivel de significancia de 0,00 $<0,05$. Por lo tanto, una buena gestión de la imagen de la marca Perú tendrá como resultado una influencia significativa en la oferta y demanda turística.

Por otro lado, no existe influencia o incidencia entre la imagen de la marca Perú y la calidad de vida para el turista de Lima Metropolitana en el año 2020, lo cual se demostró a través de los resultados un nivel de significancia de 0,220 > 0,005. Gestionar una buena imagen de la marca Perú no influirá para que el turista limeño pueda tener una buena calidad de vida.

Finalmente, se identificó una influencia significativa entre la imagen de la marca Perú y la infraestructura turística, demostrado a través de un nivel de significancia de $0,00<0,05$. Esto significa que el buen trabajo y la buena gestión de la imagen de 
la marca Perú podrá propiciar una infraestructura turística adecuada para el turista.

Se puede concluir que la imagen de la marca Perú sí resulta relevante en la generación de valor para el turista tanto de manera nacional como internacional. Una buena gestión de marca país incentiva la afluencia turística nacional, pero debe mejorarse también la oferta e infraestructura turística, lo que genera valor percibido por el cliente.

\section{Referencias}

Aguilar, M., y Arteaga, D. (2017). La generación de valor para los accionistas de las MIPYMES de la Provincia de El Oro. Guayaquil. Tesis de maestría. Universidad Espíritu Santo. http: //repositorio.uees.edu.ec/bitstream/ 123456789/1623/1/La\%20generaci\% C3\%B3n\%20de\%20valor.pdf

Aguilar, V., y Herrera, A. (2018). La supervivencia de las agencias de viaje y turismo: gestión del marketing digital como estrategia para la atracción de clientes turistas. Tesis de grado. Pontificia Universidad Católica del Perú. http://tesis.pucp.edu.pe/repositorio/ handle/20.500.12404/13122

Alba, T (2017). Cómo lograr un mayor reconocimiento de marca haciendo email marketing. [Mensaje en un blog]. Mail Relay. https://blog.mailrelay.com/es/ 2017/04/11/reconocimiento-de-marca

Angulo, D. (2019). Infraestructura truristica y oportunidades socieconómicas de la quinta el pedregal en la parroquia balsapamba, cantón san miguel provincia de Bolivar. Babahoyo. Tesis de grado. Universidad Técnica De Babahoyo. http://dspace.utb.edu.ec/bitstream/ handle/49000/5980/E-UTB-FCSEHTURIS-000057.pdf?sequence $=1 \&$ isAllowed $=\mathrm{y}$

Arce, A., y Bustamante, L. (2016). Propuesta de un diseño de infraestructura turística para la laguna de chocar. Tesis de grado. Universidad del Azuay. http://dspace.uazuay.edu. ec/handle/datos/6973

Ardila, R. (2003). Calidad de vida: una definición integradora. Revista Latinoamericana de Psicología. 35(2),161164. https://www.redalyc.org/articulo. oa?id=80535203

Ayala, N., y Reyes, A. (2019). Estudio sobre la marca país Colombia su evolución desarrollo y su relación con la imagen del país del año 2005 al 2018. Bogotá, Colombia. Tesis de grado. Universidad del Bosque. https://repositorio.unbosque.edu.co/ bitstream/handle/20.500.12495/3072/ Ayala_Montoya_Natalia_2019.pdf? sequence $=1 \&$ isAllowed $=\mathrm{y}$

Cáceres, M., y Delgado, V. (2019). El uso de la marca Perú para incentivar la oferta turística de nuestro país durante el año 2018 Lima. Tesis de grado. Universidad Tecnológica del Perú. https://repositorio.utp.edu. pe/handle/20.500.12867/2832

Carballa, C. (2016). Diferencia RSE. Reporte de caso-Howard Johnson. Tesis de grado, Universidad Siglo 21. https:// repositorio.uesiglo21.edu.ar/bitstream/ handle/ues21/17789/CARBALLA\% 20MEUCCI\%20CONSTANZA\% 
20MAGALI.pdf?sequence $=1$

Caycho, D., y Pietro, M. (2018). El efecto de la Marca Perú sobre el sector turístico de Lima en el periodo 2014 - $201 \%$. Tesis de grado. Universidad Tecnológica del Perú). http://repositorio.utp.edu. pe/bitstream/UTP/1906/1/Diana\%

20Caycho_Naysha\%20Prieto_Trabajo\% 20de\%20Investigacion_Bachiller_2018. pdf

Cotrina, G. (2018). Marca Ciudad para mejorar la promoción turística del distrito de Tarapoto. Universidad Mayor de San Marcos. http: //repositorio.unsm.edu.pe/bitstream/ handle/11458/3298/TURISMO\%20\%20Greysi\%20Samantha\%20Cotrina\% 20 Coral.pdf? sequence $=1 \&$ isAllowed $=y$

Escudero, C, y Cortéz, L. (2018). Técnicas y métodos cualitativos para la investigación científica. Editorial UTMACH. http://repositorio.utmachala.edu. ec/bitstream/48000/14207/1/Cap.1Introducci $\%$ C3\%B3n $\% 20 a \% 20 l a \%$ 20investigaci\%C3\%B3n\%20cient\%C3\% ADfica.pdf

Ferrán, Y., Naranjo, M., y Marrero, M. (2016). La marca país (2010-2014): Ecuador Ama la Vida y su incidencia en la matriz productiva y el plan del Buen Vivir. Revista UNIANDES Episteme. 3(2), 222-240. https://dialnet.unirioja. es/servlet/articulo?codigo $=6756412$

FutureBrand y Country Brand Index (2019) FutureBrand, https:// www.futurebrand.com/uploads/FCI/ FutureBrand-Country-Index-2019.pdf

Gestión (2019). Indecopi busca registrar la Marca Perú en el mundo para evitar su uso inescrupuloso. https: //gestion.pe/economia/marca-peruindecopi-indecopi-busca-registrar-lamarca-peru-en-el-mundo-para-evitarsu-uso-inescrupuloso-noticia/?ref=gesr

Hernández, R., Fernández, C., y Baptista, M. (2014). Metodología de la Investigación. Editorial McGraw Hill.

Luyo, M. (2019). La marca Perú y su incidencia en la promoción de la oferta turística en nuestro país durante el año 2017; analizando su posicionamiento y consolidación en el mercado internacional. Tesis de grado. Universidad Tecnológica del Perú. http://repositorio. utp.edu.pe/bitstream/UTP/2350/ 1/Marisol\%20Luyo_Trabajo\%20de\% 20Investigacion_Bachiller_2019.pdf

Maram, L. (2018). Reputación de marca ¿qué es y cómo construirla? Online y offline. Luis Maram, comunicamos marcas que inspiran. https://www. luismaram.com/reputacion-de-marcaque-es-y-como-construirla-online-yoffline/\#Que_es_reputacion_de_marca

Matta, R. (2012). Cocinando una Nación de Consumidores: El Perú como marca global. Consensus. 17(1), 4960. http://revistas.unife.edu.pe/index. $\mathrm{php} /$ consensus/article/view/935

Mendivez, Y (2020). Marca Perú ¿patrimonio cultural o estrategia comercial?. Actas de Diseño $N^{\circ}$ 31. XV Semana Internacional de Diseño en Palermo 2020. 178-181. Editorial Universidad de Palermo. https://fido.palermo. edu/servicios_dyc/publicacionesdc/ actas_de_diseno/detalle_articulo.php? id_libro $=807 \&$ id_articulo $=17215$ 
Mero, R. (2015). El tipo de infraestructura turística estacional y su incidencia en el flujo de turistas del sector urbano del cantón Puerto Lopéz. Tesis de grado, Universidad Estatal Del Sur De Manabí. http://repositorio.unesum.edu. ec/bitstream/53000/631/1/UNESUMECU-ECOT-2015-22.pdf

Mori, M. (2018). Generación de valor público en el sector turismo, Tarapoto. Tesis de grado. Universidad Nacional de San Martín. http: //repositorio.unsm.edu.pe/bitstream/ handle/11458/2641/TURISMO\%20\%20Marilyn\%20Mori\%20Ru\%c3\%adz. pdf? sequence $=1 \&$ isAllowed $=y$

Mousalli, G. (2015). Métodos y diseños de investigación cuantitativa. Revista Researchgate. https://www.researchgate.net/ publication/303895876_Metodos_y_ Disenos_de_Investigacion_Cuantitativa

Otzen, T., y Manterola, C. (2017). Técnicas de Muestreo sobre una Población a Estudio. International journal of morphology. 35(1), 227232. https://scielo.conicyt.cl/pdf/ ijmorphol/v35n1/art37.pdf

Promperú (2018). Libro de Oro de la Marca Perú. Grupo Skateholders S.A.C. https://issuu.com/stakeholdersrs/ docs/libro_marca_per__2018

PROMPERÚ (2020). Conozca los lineamientos de transporte aéreo de pasajeros para la prevención del COVID-19 publicado por el MTC. $Y$ tú qué planes. https://ytuqueplanes. com/volver/\#/comunicados/prevenirpara-volver-a-volar
Ricaurte, C., y Juan, A. (2016). Marca País: Análisis retrospectivo y retos a futuro para Colombia. Tesis de maestría. Colegio de estudios superiores de Administración. http://hdl.handle. net/10726/1517

Robles, A., Rubio, B., De la Rosa, E., y Nava, A. (2016). Generalidades y conceptos de calidad de vida en relación con los cuidados de la salud. El residente. 11(3), 120-125. https://www.medigraphic.com/pdfs/ residente/rr-2016/rr163d.pdf

Roldán, P., y Fachelli, S. (2015). Metodología de la investigación social cuantitativa. Recuperado de https://ddd.uab.cat/pub/caplli/2017/ 185163/metinvsoccua_cap2-4a2017.pdf

Romero, M. (2016). Productos, servicios y destinos turísticos HOTG0108. IC Editorial.

Sánchez, A (2019). El proceso de creación de "Más Peruano Que" y de sus piezas publicitarias: una campaña de Marca Perú basada en generar orgullo peruano. Tesis de grado. Pontificia Universidad Católica del Perú. http://tesis.pucp.edu.pe/ repositorio/bitstream/handle/20.500. 12404/9133/SANCHEZ_JAUREGUI_ EL_PROCESO_DE_CREACION_ DE_MAS_PERUANO_QUE.pdf? sequence $=6 \&$ is Allowed $=\mathrm{y}$

Sancho, A. (2016). Introducción al turismo. Publicado por Organización Mundial del Turismo.http: //www.utntyh.com/wpcontent/ uploads/2011/09/INTRODUCCIONAL-TURISMO-OMT.pdf 
Secretaría General de Turismo (2007). Plan de Turismo Español Horizonte 2020. Documento Ejecutivo. http: //www.minetur.gob.es/turismo/eses/turismo2020/paginas/turismo2020. aspx

Suarez, F. (2019). La reputación y la imagen en empresas y marcas. Consultoría Estratégica de Investigación de Mercado. https://www.cimec.es/reputacionimagen-empresas-marcas/

Sulz, P. (2019). Branding: aprende cómo hacer una gestión de marca increíble. Rockcontent. https://rockcontent.com/ es/blog/branding/ 